\title{
Radio Wave Satellite Propagation in Ka/Q Band
}

\author{
László Csurgai-Horváth*, Bernard Adjei-Frimpong1, Carlo Riva², Lorenzo Luini² \\ ${ }^{1}$ Department of Broadband Infocommunications and Electromagnetic Theory, Faculty of Electrical Engineering and Informatics, \\ Budapest University of Technology and Economics, H-1111 Budapest, Egry J. u. 18, Hungary \\ 2 DEIB, Politecnico di Milano and IEIIT, CNR, Piazza Leonardo da Vinci 32, 20133 Milano, Italy \\ * Corresponding author, email: csurgai@hvt.bme.hu
}

Received: 23 May 2017, Accepted: 12 March 2018, Published online: 23 May 2018

\begin{abstract}
In 2013 the European Space Agency, in cooperation with Inmarsat, launched the Alphasat communication satellite hosting four Technology Demonstration Payloads (TDPs). One of them is the Aldo Paraboni payload, supported by the Italian Space Agency (ASI) and executed by ESA in the framework of the Advanced Research in Telecommunications Systems (ARTES) 8 Telecom program. In addition to the Communication experiment, it includes the Alphasat Scientific Experiment transmitting coherent beacon signals at Ka-band $(19.701 \mathrm{GHz})$ and Q-band $(39.402 \mathrm{GHz})$. The satellite supports a Europe-wide experiment to investigate the atmospheric propagation effects occurring in Ka and $\mathrm{Q}$ bands. The demand for increasing bandwidth in the satellite radio communication domain is moving the communication channels to the higher frequency bands. Hence for both research and commercial purposes is especially important to effectively explore the $\mathrm{Q}$ band that is affected by attenuation, depolarization and scintillation due to different atmospheric effects. In 2014 the Department of Broadband Infocommunications and Electromagnetic Theory at Budapest University of Technology and Economics joined the ASAPE (AlphaSat Aldo Paraboni Experimenters) group and developed a ground station to be installed in Budapest. This work was supported by the European Space Agency under its Plan for European Cooperating States program. Our paper gives the background of the Alphasat Scientific Experiment and overviews the design phases of the receiver station in Budapest. We present also the processing and validation of data recorded so far and our future experimenting plans.
\end{abstract}

Keywords

Ka-band, Q-band, attenuation statistics, Alphasat

\section{Introduction}

New broadband satellite communication services require high speed broadband channels. As the frequency bands below $15 \mathrm{GHz}$ become more and more crowded there is an increasing demand for using higher frequency bands. However, at these frequencies there are special challenges considering the higher impacts of the atmospheric impairments. This necessitates the comprehensive characterization of the channel, based on statistical analysis of measurements. In order to investigate the radio propagation above Ka-band, ASI conceived and supported [1, 2] a new experiment executed by ESA through the realization of the Aldo Paraboni payload on board of the Alphasat satellite, launched on July 25, 2013. The experiment allows a measurement campaign over Europe investigating the propagation effects in Ka and Q-band. The community of experimenters, joining the ASAPE (AlphaSat Aldo Paraboni Propagation Experimenters) group, is operating several receiver stations, typically equipped with meteorological and radiometric devices [1-9].

The Department of Broadband Infocommunications and Electromagnetic Theory at Budapest University of Technology and Economics (BME-HVT) designed and installed a receiver station for both beacons at 19.701 and $39.402 \mathrm{GHz}$ that was operative in 2014 [7]. Minor modifications were then applied to maximize the receiver performance.

The structure of this paper is the following: Section 2 gives the overview of the Alphasat Aldo Paraboni Experiment from the perspective of the Principal Investigator (PI). Section 3 introduces the Group of the AlphaSat Aldo Paraboni propagation Experimenters (ASAPE). Section 4 presents the beacon receiver station in Budapest, while Section 5 is an overview of the relevant ITU-R regulations to estimate the rain attenuation statistics 
for satellite connections. Finally, Section 6 includes measured annual statistics compared with the estimations that can be calculated using the ITU-R models. The paper ends with conclusions and acknowledgement.

\section{The Alphasat Aldo Paraboni Scientific Experiment}

The propagation community spent a great effort, since the 70 s to the end of the last century, to set up satellite propagation experiments, which investigates atmospheric effects at satellite frequency bands. The USA and Japan realized satellite propagation experiment at $\mathrm{Ku}$ and $\mathrm{Ka}$ band in the 70 s and the 90s. In Europe, the Italian Sirio and Italsat, and the ESA Olympus satellites hosted propagation payloads at $\mathrm{Ka}$ and $\mathrm{Q} / \mathrm{V}$ bands in the same period. These experimental campaigns were followed by intense modelling activities, bringing to the development of several models, some of which were accepted as standard of the ITU-R (International Telecommunication Union - Radiocommunication Sector). Nevertheless, some issues needed deeper investigation; among them, few results were available above the Ka band (only with the Italsat experiment); due to the reduced receivers' dynamic range significant attenuation events were not recorded; the standard sampling rate (1 sample per second) was not adequate for a complete characterization of the signal spectrum (scintillation) and few measurements of XPD (cross polar discrimination) were collected. Finally, the quite limited period of measurements simultaneously available in several European sites did not allow a good characterization of spatial correlation of various atmospheric effects.

The new Aldo Paraboni propagation experiment has been conceived to address most of these issues. In fact, few years ago, the ESA Alphasat program offered the opportunity to host Technological Demonstration Payloads on board of the Alphasat commercial satellite operated by Inmarsat. One of the selected proposals was the Aldo Paraboni Payload [1]. The Aldo Paraboni payload is composed by the communication experiment (COMEX) payload in $\mathrm{Q} / \mathrm{V}$ band and by the "scientific" (propagation) experiment (SCIEX) payload, in Q and Ka bands, covering the whole Europe. The payload development was supported by the Italian Space Agency (ASI) as in-kind contribution to the Alphasat project, which is executed by the European Space Agency (ESA) in the framework of the ARTES 8 Telecom program $[10,11]$. The Alphasat satellite was successfully launched on July $25^{\text {th }}$, 2013 and it flies along a geosynchronous orbit with an inclination above the Equatorial plane not exceeding 3 degrees.

The Aldo Paraboni propagation payload consists of two coherent beacons at 19.701 and $39.402 \mathrm{GHz}$, as shown in
Fig. 1. The main characteristics of the beacons are listed in Table 1, while the coverage areas are plotted in Fig. 2.

\section{Group of the AlphaSat Aldo Paraboni propagation Experimenters}

Due to the important investment by Italy on the Alphasat Aldo Paraboni experiment, a large international participation is highly encouraged by ASI and ESA. In fact, the experiment is expected to provide the scientific community with new experimental data allowing the validation, among the others, of models of space-time correlation of rain/attenuation fields, of site diversity at small and large scale, and of spatial correlation of cloud fields. All these developments shall contribute to radio regulations and support the implementation of new satellite communication systems.

In order to achieve these objectives, a strong coordination of the experimenters is essential, as demonstrated by previous projects (COST 205 project on OTS and Sirio satellites, the ESA OPEX for Olympus, the NASA NAPEX for ACTS,

Table 1 Main characteristics of scientific experiment beacons

\begin{tabular}{llc}
\hline & Frequency & $19.701 \mathrm{GHz}$ \\
Ka band beacon & Polarization & Linear V \\
& Antenna Boresight & $32.5^{\circ} \mathrm{N}, 20^{\circ} \mathrm{E}$ \\
& EIRP & $19.5 \mathrm{dBW}$ \\
\hline \multirow{3}{*}{ Q band beacon } & Frequency & $39.402 \mathrm{GHz}$ \\
& Polarization & Linear tilted $45^{\circ}$ \\
& Antenna Boresight & $45.4^{\circ} \mathrm{N}, 9.5^{\circ} \mathrm{E}$ \\
& EIRP & $26.5 \mathrm{dBW}$ \\
\hline
\end{tabular}

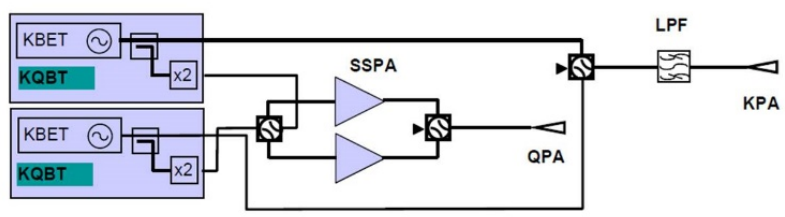

Fig. 1 Block Diagram of the Alphasat Aldo Paraboni Scientific Experiment Payload (courtesy of Thales Alenia Space - Italy)

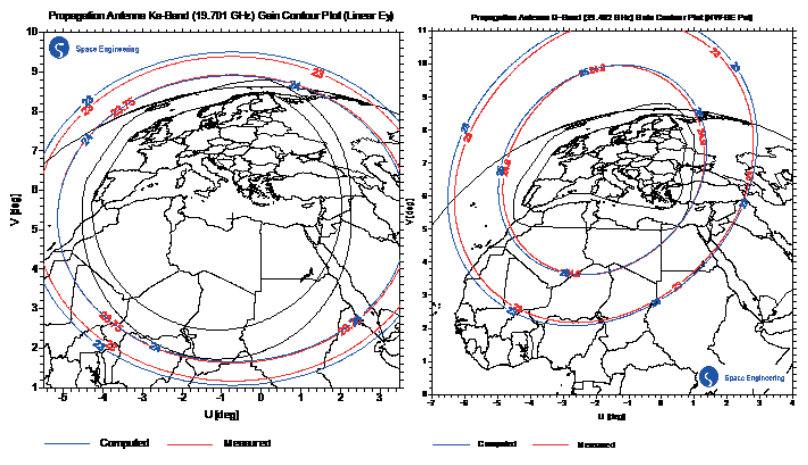

Fig. 2 Coverage areas of Ka Band (a) and Q Band (b) beacons 
the ASI CEPIT for ITALSAT and, more recently, the COST IC0802 for Ka band campaigns [12]) and by contributions to ITU-R Study Group 3, Radiowave Propagation, and ITUR-P recommendations for SatCom systems.

To this aims, in 2014, ASI and ESA promoted the constitution of the collaborative Group of the AlphaSat Aldo Paraboni propagation Experimenters (ASAPE), which is an open forum of researchers performing propagation campaigns with the Aldo Paraboni payload and other satellite payloads at $\mathrm{Ka}$ band. Topics include: instruments, design and execution of campaigns, data analysis, use of remote sensing and meteorological data and use of numerical weather products. The group also aims to be a reference for the use of measurements to foster model development and theoretical advances, as well as to actively pursue transfer of results to industry and into radio regulations. The group has collected the participation to the measurement campaign by several experimenters with different receivers. Table 2 shows the list of the current receiving ground stations in Europe, whose location is also reported in Fig. 3.

Table 2 List of receiving stations for the Alphasat Aldo Paraboni propagation experiment

\begin{tabular}{|c|c|c|c|}
\hline Site & Country & $\begin{array}{c}\text { Latitude } \\
(\text { deg. N) }\end{array}$ & $\begin{array}{c}\text { Longitude } \\
\text { (Deg. E) }\end{array}$ \\
\hline Spino d'Adda & Italy & 45.40 & 9.50 \\
\hline Tito Scalo & Italy & 45.40 & 9.50 \\
\hline Roma & Italy & 41.83 & 12.47 \\
\hline Milano & Italy (NASA) & 45.50 & 9.20 \\
\hline Graz & Austria & 47.09 & 15.46 \\
\hline $\begin{array}{l}\text { Louvain-la- } \\
\text { Neuve }\end{array}$ & Belgium & 50.40 & 4.37 \\
\hline Prague & Czech Rep. & 50.04 & 14.49 \\
\hline Toulouse & France & 43.57 & 1.47 \\
\hline $\begin{array}{l}\text { Salon De } \\
\text { Provence }\end{array}$ & France & 43.62 & 5.12 \\
\hline Athens & Greece & 37.97 & 23.78 \\
\hline Lavrion & Greece & 37.72 & 24.05 \\
\hline Budapest & Hungary & 47.48 & 19.06 \\
\hline Aveiro & Portugal & 40.62 & -8.63 \\
\hline Ljubljana & Slovenia & 46.04 & 14.49 \\
\hline Madrid & Spain & 40.50 & -3.70 \\
\hline Vigo & Spain & 42.23 & -8.71 \\
\hline $\begin{array}{l}\text { Robledo de } \\
\text { Chavela }\end{array}$ & Spain (NASA) & 40.43 & -4.25 \\
\hline Chilbolton & U. K. & 51.15 & -1.43 \\
\hline Chilton & U. K. & 51.57 & -1.29 \\
\hline Edinburgh & U. K. & 55.91 & -3.32 \\
\hline
\end{tabular}

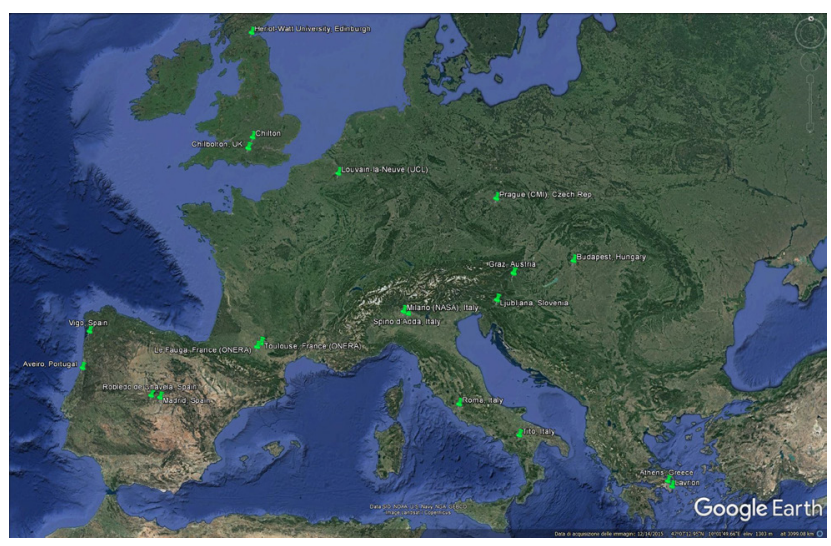

Fig. 3 Maps of the Alphasat Aldo Paraboni receiving stations.

The group held regular General Assemblies on October 2014, 2015 and 2016, during the $\mathrm{Ka}$ and Broadband Communications Conference [13]. In addition, since 2015, the Group organises periodic intermediate meetings, and a Work Group and programmatic Workshop tool place in Erice in October 2016.

\section{Alphasat beacon receiver in Budapest}

The receiver station is installed on the flat roof of the department's building at $120 \mathrm{~m}$ above sea level with $47.48^{\circ} \mathrm{N}$ latitude and $19.06^{\circ} \mathrm{E}$ longitude coordinates. As the orbit inclination of the satellite can reach $3^{\circ}$, the receiver is equipped with step-tracking system to track the satellite. Fig. 4 depicts the schematic block diagram of the system, showing the antennas with the tracking mechanism, outdoor and indoor units and the supplemental units like the weather station and power supplies:

The Cassegrain antennas [19], are equipped with the Out-Door Units (ODU) on the back of the reflector dishes. The High Performance Antennas are originally manufactured for the backbone network of terrestrial mobile

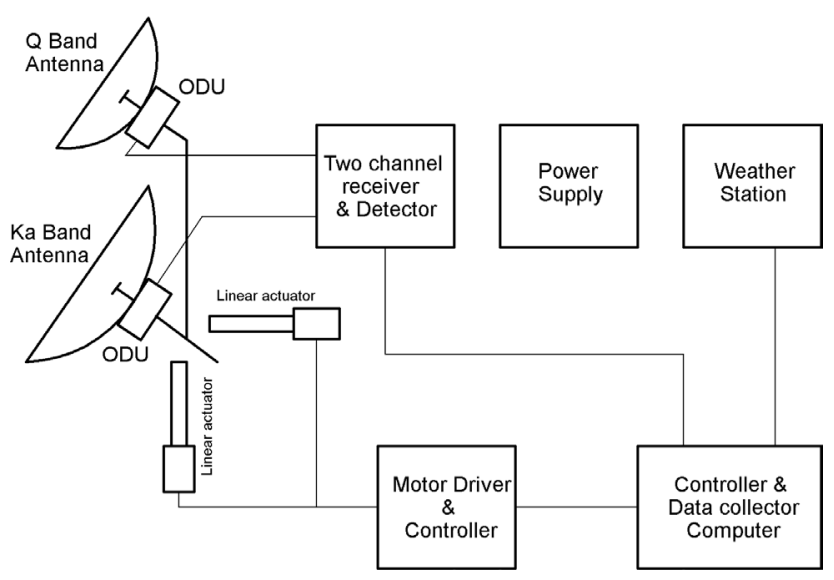

Fig. 4 Block scheme of the Alphasat two channel beacon receiver 
radio systems, but considering their high interference protections and excellent radiation characteristics they are appropriate to receive the $\mathrm{Ka}$ and $\mathrm{Q}$-band beacon frequencies of the Alphasat satellite. Fig. 5 depicts the complete outdoor system.

The antennas are manufactured by GRANTE Antenna Development and Production Corporation. We selected a $0.3 \mathrm{~m}$ antenna for Q-band and a $0.6 \mathrm{~m}$ antenna for Ka-band that have similar gain and half-power beam widths and ensure almost equal received signal power on IF band.

In terrestrial communications point-to-point microwave radios are applied to interconnect cellular network elements. As Ka and Q frequency bands are commonly used for those networks, similar devices can be easily employed as satellite receiver with some modifications. Hence our beacon receiver is based on the ODU construction of Totaltel [20]. The radio is a double conversion heterodyne receiver with synthesized local signal sources. In order to build a low-noise system with high fade margin the noise figure of the LNA block in the ODU is improved to $3 \mathrm{~dB}$. To generate a stable and jitter-free down-converted intermediate frequency (IF) signal, the reference oscillator in the ODU is a low phase noise oven-controlled crystal oscillator (OCXO) with less than $\pm 1.0 \mathrm{ppb}$ /day stability (Fig. 6).

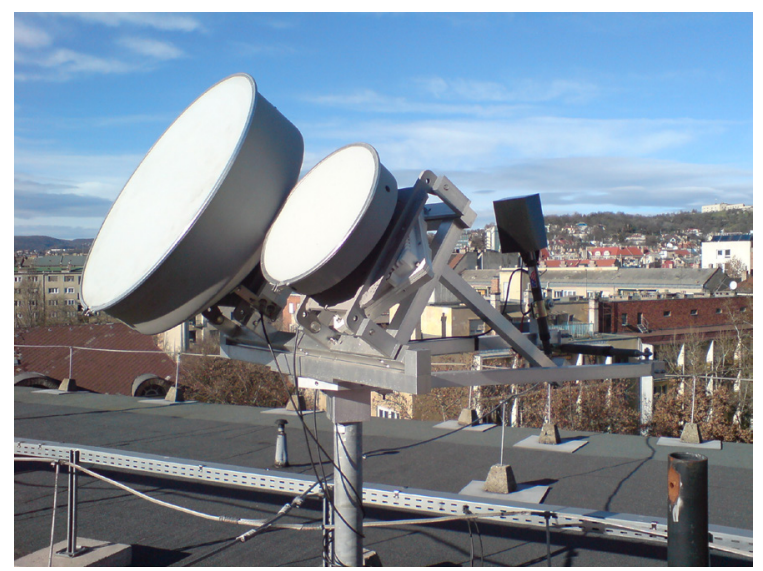

Fig. 5 High performance antennas and the tracking system

Table 3 Antenna parameters

\begin{tabular}{lcc}
\hline & Ka-band & Q-band \\
\hline Type (Cassegrain) & HPA 0.6 S 180230 & HPA 0.3 S 380 SR \\
Frequency range & FR & $37-39.5 \mathrm{GHz}$ \\
Diameter & $0.6 \mathrm{~m}$ & $0.3 \mathrm{~m}$ \\
Mid band gain & $39.5 \mathrm{dBi}$ & $39.2 \mathrm{dBi}$ \\
Polarization & Linear simplex $(\mathrm{V} / \mathrm{H})$ & Linear simplex $(\mathrm{V} / \mathrm{H})$ \\
$\begin{array}{l}\text { Half-power beam } \\
\text { width }\end{array}$ & $1.6^{\circ}$ & $1.7^{\circ}$ \\
\hline
\end{tabular}

The down converted to $100 \mathrm{kHz}$ bandwidth limited and amplified IF signal at $140 \mathrm{MHz}$ is connected with a low attenuation coaxial cable to the In-Door Unit (IDU). The calibrated gain of the ODU is $100 \mathrm{~dB}$, while the interconnecting cable between the IDU and ODU provides the supply voltage for the outdoor unit. The downlink IF transfers also modulated subcarriers of a duplex control/ telemetry channel that informs about the current system status and provides temperature information allowing the IDU to compensate the temperature caused variations of the ODU's gain. Therefore the system can measure precisely the received signal power.

The IDU (Fig. 7) is based on a modified I-Q demodulator that processes the incoming IF signal. The $140 \mathrm{MHz}$ IF signal is under sampled with an $80 \mathrm{MHz}$ analog/digital converter unit, thus further functions are implemented by digital signal processing. A quadrature digital down

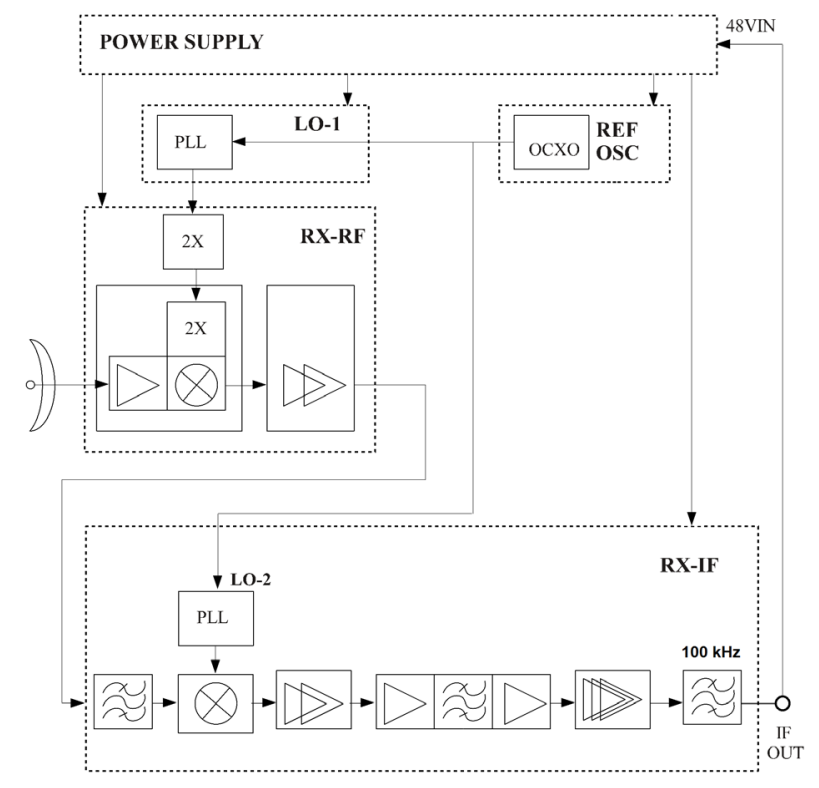

Fig. 6 Block diagram of the Out-Door Unit

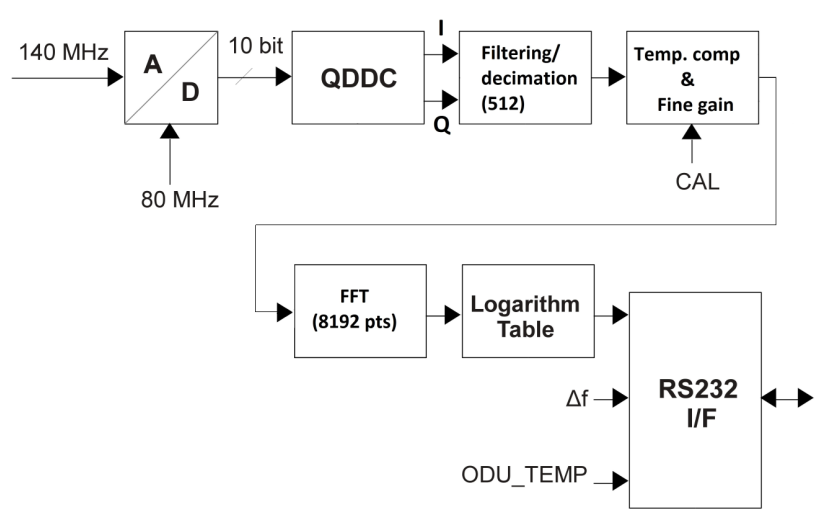

Fig. 7 Block diagram of the In-Door Unit 
converter (QDDC) module converts down the sampled signal into baseband quadrature component (I, Q) signals. The baseband signals are decimated by a factor of 512, filtered before performing the 8192 point FFT.

The beacon signal is detected as the spectral component with highest amplitude. The signal amplitude measurement is performed with $0.2 \mathrm{dBm}$ resolution; the values are forwarded with 1 sample/second rate to the data collecting system.

By taking into account the speed of A/D conversion, the decimation and the FFT buffer size, the FFT bin size is $19.07 \mathrm{~Hz}$. In order to smooth the fast signal fluctuation, 3 bins are averaged, therefore we calculate with $59 \mathrm{~Hz}$ signal bandwidth. The noise figure and bandwidth of the receiver allows detecting signals in the range of -100 to -150 $\mathrm{dBm}$. Two independent, identical processing units are performing a simultaneous $\mathrm{Ka}$ and Q-band beacon detection.

To qualify the beacon receiver and estimate the highest detectable attenuation at the receiving location, a link budget was calculated. The parameters required for that calculation are detailed in Table 4.

The link budget calculation was performed according to the following steps. Equation (1) gives the free space attenuation where $d$ is the Earth-satellite distance and $f$ is the transmit frequency:

$A_{f s}=32.44+20 \log f^{(M H z)}+20 \log d^{(k m)}(d B)$.

With Eq. (2) the noise temperature of the Low Noise Amplifier (LNA) is calculated, where $T_{0}$ is the environmental temperature $\left(290^{\circ} \mathrm{K}\right.$ was considered $), N F$ is the Noise Figure of the LNA given by the manufacturer:

$T_{L N A}=T_{0}\left(10^{N F / 10}-1\right)(K)$.

We approximate the system noise temperature (3) with the sum of the LNA's and the antenna's noise temperature:

$T_{s y s}=T_{a n t}+T_{L N A}(K)$.

In Eq. (4) $G / T$ qualifies the system where $G_{R X}$ is the gain of the receiver antenna and $T_{s y s}$ is the system noise temperature:

$G / T=G_{R X}-10 \log \left(T_{s y s}\right)(d B / K)$.

The noise power is expressed with Eq. (5) where $k$ denotes the Boltzmann-constant, $T_{s y s}$ is the system noise temperature and $B$ is the receiver bandwidth.

$N_{p}=10 \log \left(k T_{s y s} B\right)(d B W)$.

$C_{p}$ is the carrier power (6) calculated from the Effective Isotropically Radiated Power (EIRP), the free space loss
Table 4 Parameters for link budget calculation

\begin{tabular}{|c|c|c|c|}
\hline & Ka-band & Q-band & \\
\hline Frequency & 19.701 & 39.402 & $\mathrm{GHz}$ \\
\hline Guaranteed EIRP & 19.5 & 26.5 & dBW \\
\hline Earth-satellite distance & 35756.0 & 35756.0 & $\mathrm{~km}$ \\
\hline Free-space attenuation & 209.4 & 215.4 & $\mathrm{~dB}$ \\
\hline $\begin{array}{l}\text { Aggregate non-rain } \\
\text { attenuation }\end{array}$ & 2.0 & 2.0 & $\mathrm{~dB}$ \\
\hline Receiving antenna gain & 39.5 & 39.2 & $\mathrm{~dB}$ \\
\hline Receiver noise figure & 3.0 & 3.0 & $\mathrm{~dB}$ \\
\hline Antenna noise temperature & 25.0 & 25.0 & K \\
\hline LNA Noise Temperature & 288.6 & 288.6 & $\mathrm{~K}$ \\
\hline Receiver system G/T & 14.5 & 14.2 & $\mathrm{~dB} / \mathrm{K}$ \\
\hline Received carrier power & -152.4 & -151.7 & $\mathrm{dBm}$ \\
\hline $\begin{array}{l}\text { Carrier to noise power } \\
\text { density } \mathrm{C} / \mathrm{N}_{0}\end{array}$ & 53.2 & 53.9 & $\mathrm{dBHz}$ \\
\hline Receiver bandwidth & 59 & 59 & $\mathrm{~Hz}$ \\
\hline Noise power & -185.8 & -185.8 & dBW \\
\hline $\mathrm{C} / \mathrm{N}$ & 33.5 & 34.1 & $\mathrm{~dB}$ \\
\hline $\mathrm{C} / \mathrm{N}_{\min }$ & 4.4 & 4.4 & $\mathrm{~dB}$ \\
\hline Rain margin & 29.1 & 29.8 & $\mathrm{~dB}$ \\
\hline
\end{tabular}

$A_{f s}$, the aggregated non-rain attenuation factors $\left(A_{a}\right)$ and the receiver antenna gain $G_{R X}$ :

$C_{p}=E I R P-A_{f s}-A_{a}+G_{R X}(d B W)$.

The carrier to noise power density (7) can be expressed with the following difference:

$C / N_{0}=E I R P-A_{f s}+G / T-k(d B H z)$.

The carrier to noise ratio (8) is the difference of the carrier and the noise power:

$C / N=C_{p}-N_{p}(d B)$.

Finally, to calculate the rain margin (9) the value of the smallest detectable carrier to noise ratio $C / N_{\min }$ should be subtracted from $C / N$ :

$R_{m}=C_{p}-N_{p}-C / N_{\min }(d B)$.

This is the dynamics of the receiver and determines the highest attenuation that can be detected with the system.

\section{Model of first order attenuation statistics}

There are several effects that may cause attenuation on the Earth-space propagation channel. The ITU-R P.618 recommendation [14] is a comprehensive description of these effects and of the related calculations. The main source of atmospheric attenuation is rain. ITU-R P.618 recommends 
a model to estimate the long-term attenuation statistics based on the satellite and receiver system parameters. The first order statistics provided by the model can be preliminary used for a sort of validation of the measured time series and also to reveal local microclimate or model inaccuracy if a noticeable difference exists between the model and the measurements. Other ITU-R recommendations as ITU-R P.837 [15], P.838 [16], P.839 [17], and P.678 [18] are supporting these calculations. The ITU R model permits calculation of annual path attenuation statistics at different geographical locations for frequencies up to $55 \mathrm{GHz}$. After the model description, a comparative study will be provided to validate the measurements in Budapest in the Ka and Q-band.

The ITU model works with the following main input parameters:

$R_{001}$ : point rainfall rate for the location exceeded for $0.01 \%$ of an average year $(\mathrm{mm} / \mathrm{h})$

$h_{s}$ : earth station height above mean sea level $(\mathrm{km})$

$\varphi$ : latitude of the Earth station (degrees)

$\theta$ : satellite elevation angle (degrees)

$f$ : satellite receiving frequency $(\mathrm{GHz})$

$R_{e}$ : effective radius of the Earth $(8500 \mathrm{~km})$.

The rain height $h_{R}$, can be determined by using the map information in recommendation ITU-R P.839 [17].

For $\theta \geq 5^{\circ}$ the slant path length $L_{s}$ can be computed with Eq. (10):

$L_{s}=\frac{\left(h_{R}-h_{s}\right)}{\sin \theta} \quad[\mathrm{km}]$.

For $\theta<5^{\circ}$, the following formula is used:

$$
L_{s}=\frac{2\left(h_{R}-h_{s}\right)}{\left(\sin ^{2} \theta+\frac{2\left(h_{R}-h_{s}\right)}{R_{e}}\right)^{1 / 2}+\sin \theta} \quad[\mathrm{km}]
$$

If $h_{R}-h_{s}$ is less than or equal to zero, the predicted rain attenuation for any time percentage is zero and the following steps are not required.

The horizontal projection of the slant path length, $L_{G}$ is determined by Eq. (12):

$$
L_{G}=L_{S} \cos \theta \quad[k m] .
$$

The long-term statistics of rainfall rate, $R_{001}$, exceeded for $0.01 \%$ of an average year (with an integration time of 1 minute) can be obtained from local data sources. If data is not available, an estimate can be obtained from the maps of rainfall rate given in Recommendation ITU R P.837 [15].
If $R_{001}$ is equal to zero, the predicted rain attenuation is zero for any time percentage and the following steps are not required.

The specific attenuation $\gamma_{R}$, can be determined with Eq. (13) by using the frequency-dependent coefficients given in ITU R P.838 [16] and the rainfall rate $R_{001}$.

$\gamma_{R}=k\left(R_{001}\right)^{\alpha} \quad[d B / k m]$.

The horizontal reduction factor $r_{001}$ for $0.01 \%$ of the time is given by Eq. (14):

$$
r_{0.01}=\frac{1}{1+0.78 \sqrt{\frac{L_{G} \gamma_{R}}{f}}-0.38\left(1-e^{-2 L_{G}}\right)} .
$$

The vertical adjustment factor $v_{001}$, for $0.01 \%$ of the time can be calculated with Eq. (15)-(18) as it follows:

$\zeta=\tan ^{-1}\left(\frac{h_{R}-h_{s}}{L_{G} r_{0.01}}\right) \quad[\mathrm{deg}]$.

For $\zeta>\theta$,

$L_{R}=\frac{L_{G} r_{0.01}}{\cos \theta}[\mathrm{km}]$

else

$L_{R}=\frac{\left(h_{R}-h_{s}\right)}{\sin \theta}[\mathrm{km}]$.

If $|\varphi|<36^{\circ}, \chi=36-|\varphi|$ degrees.

Else, $\chi=0$ degrees and $v_{001}$ is:

$v_{0.01}=\frac{1}{1+\sqrt{\sin \theta}\left(31\left(1-e^{-(\theta /(1+\chi))}\right) \frac{\sqrt{L_{R} \gamma_{R}}}{f^{2}}-0.45\right)}$.

The effective path length is the product of $\mathrm{L}_{\mathrm{R}}$ and $v_{001}$ :

$L_{E}=L_{R} v_{001} \quad[k m]$.

The predicted attenuation exceeded for $0.01 \%$ of an average year is given by Eq. (20):

$A_{001}=\gamma_{R} L_{E} \quad[d B]$.

For other percentages of an average year the estimated attenuation to be exceeded, in the range $0.001 \%$ to $5 \%$, is determined from the attenuation to be exceeded for $0.01 \%$ for an average year:

If $p \geq 1 \%$ or $|\varphi| \geq 36^{\circ}: \beta=0$.

If $p<1 \%$ and $|\varphi|<36^{\circ}$ and $\theta \geq 25^{\circ}: \beta=-0.005(|\varphi|-36)$.

Otherwise: $\beta=-0.005(|\varphi|-36)+1.8-4.25 \sin \theta$. 
The estimate of the long term statistics of attenuation due to rain is given by Eq. (21).

$A_{P}=A_{001}\left(\frac{P}{0.01}\right)^{-\left(0.655+0.033 \ln (P)-0.045 \ln \left(A_{001}-\beta(1-P) \sin \theta\right)\right.}[d B]$.

Table 5 lists the most important intermediate values obtained from the calculations above:

ITU-R P.618 is applicable to model first order attenuation statistics for satellite channels with rain attenuation. In the next section the statistics of measured attenuation time series will be compared with this model and conclusions will be drawn.

\section{Measurements}

The results presented in the following correspond to the complete year 2016. The receiver station collects the received power with 1 sample/sec rate, while the meteorological data with 1 sample/min. The following figure depicts the received power time series for both beacons together with the measured rainfall rate.

The effect of the satellite's daily movement is scarcely observed thanks to the tracking system. The diurnal variation during clear-sky conditions is usually below $\pm 0.5 \mathrm{~dB}$ that is acceptably low. Several reasons may cause it, like the temperature variation of the satellite or the receiver, the antenna mispointing, or any atmospheric effect besides rain.

July in 2016 was a rainy period as can be observed in Fig. 8, where rain events above $60 \mathrm{~mm} / \mathrm{h}$ were also detected. As the higher frequencies are more intensively affected by rain, the attenuation at Q band is always higher than at Ka-band.

For propagation modelling and statistics, the time series of attenuation provides the most relevant information. In order to convert the received power to attenuation, their measured values are subtracted from the median (clear sky) received power that is calculated on monthly basis. The observable attenuation events are mainly caused by rainy periods; a typical one is depicted in Fig. 9.

The Complementary Cumulative Distribution Function (CCDF) of rain attenuation provides the probability of exceeding different attenuation levels. The monthly

\begin{tabular}{lc}
\multicolumn{2}{c}{ Table 5 Intermediate parameters for the Budapest station } \\
\hline$R_{001}$ point rainfall rate & $42 \mathrm{~mm} / \mathrm{h}$ \\
\hline Rain height $h_{R}$ & $2.95 \mathrm{~km}$ \\
Slant path length $L_{s}$ & $4.92 \mathrm{~km}$ \\
Effective path length $L_{E}$ & $4.60 \mathrm{~km}$ \\
\hline
\end{tabular}
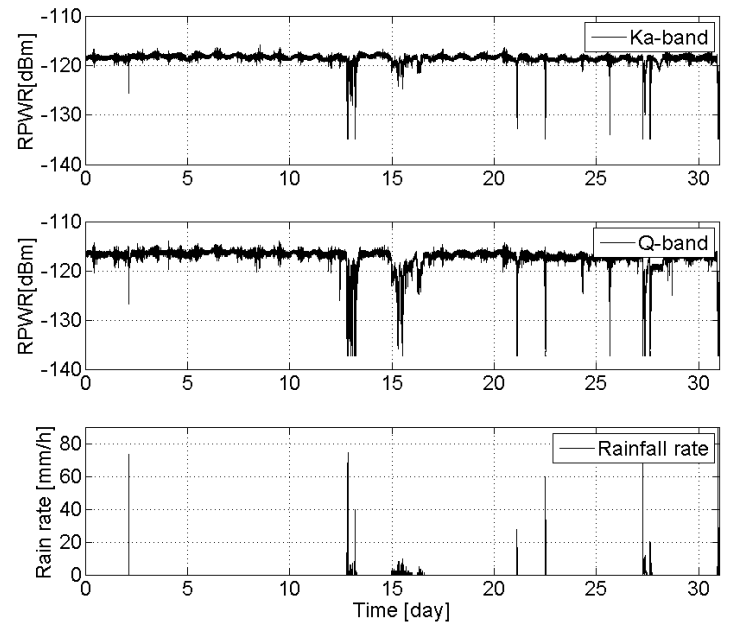

Fig. 8 Attenuation and rainfall rate (July, 2016)
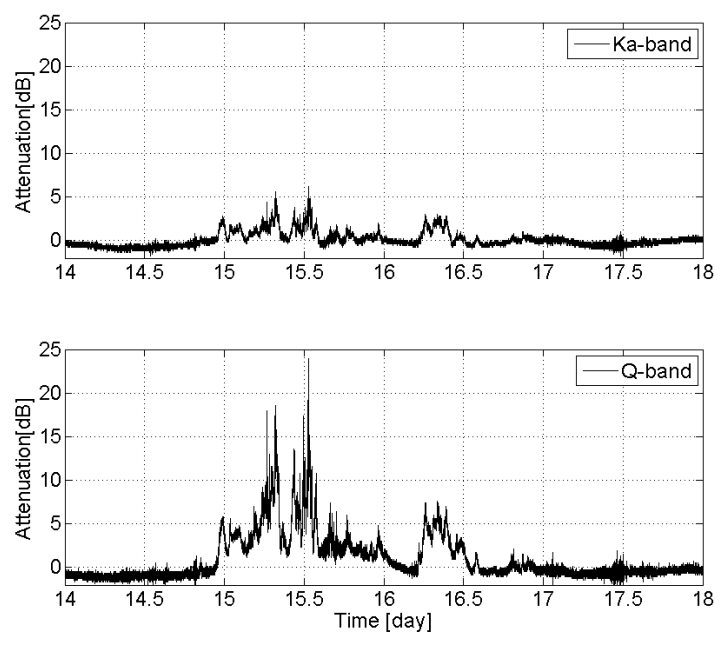

Fig. $9 \mathrm{Ka}$ and Q-band attenuation time series (14-18 July 2016.)

distributions are significantly influenced by the actual weather conditions as are depicted in Fig. 10 and Fig. 11 for the $\mathrm{Ka}$ and the Q-band channels, respectively.

Fig. 12 depicts the CCDF of the measured attenuation for $\mathrm{Ka}$ and Q-band together with the distribution curves predicted by the ITU-R P.618 recommendation.

It should be noticed that one important model parameter for rain attenuation prediction is $R_{001}$, the rain rate exceeded for $0.01 \%$ of an average year, which characterizes the local climate. According to the precipitation map provided by ITU [15], $R_{001}$ is equal to $38.1 \mathrm{~mm} / \mathrm{h}$ for Budapest. Nevertheless, this constant may be influenced by the actual annual weather conditions causing deviations from the long-term statistics. In Fig. 12 is also observable the effect of the relatively low system fade margin: there are no evaluable data above $20 \mathrm{~dB}$ for the Q-band channel. 


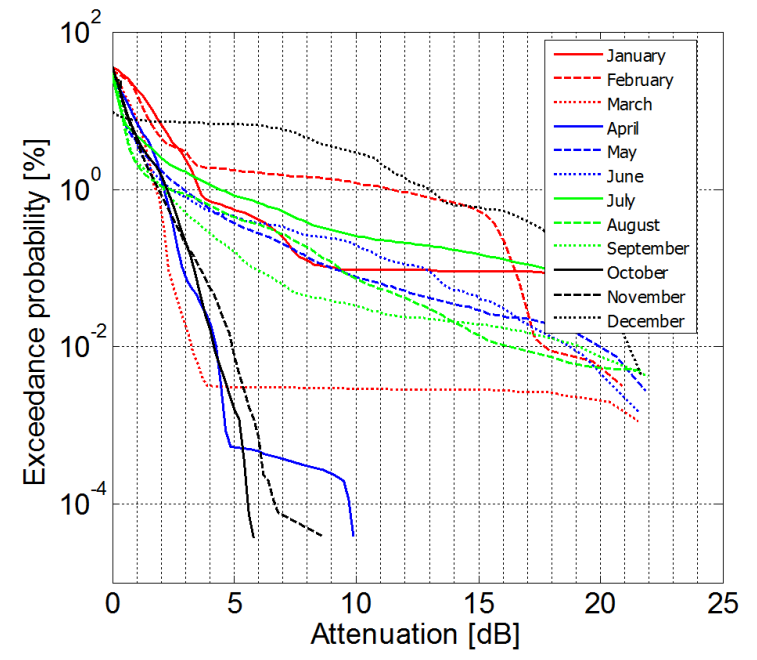

Fig. 10 CCDF of Ka and Q-band annual attenuation, 2016

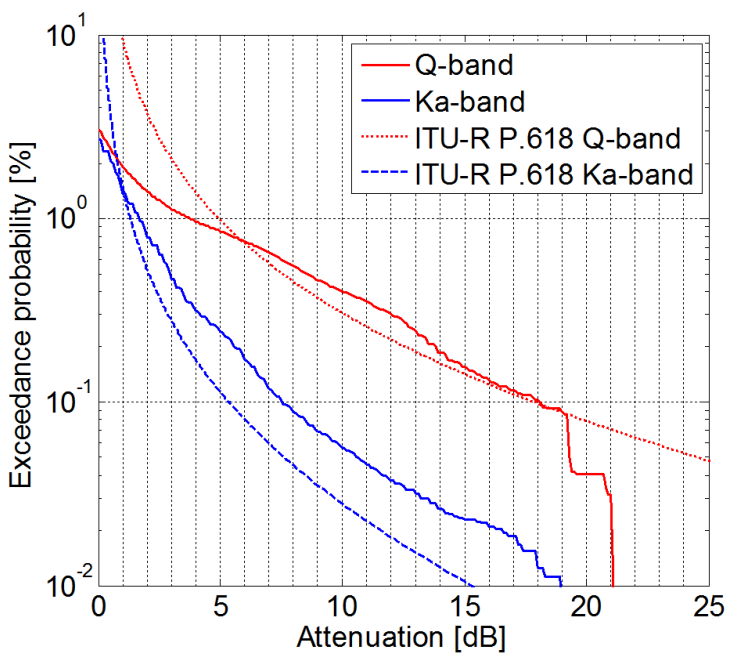

Fig. 12 CCDF of measured $\mathrm{Ka}$ and Q-band annual attenuation compared with the ITU-R P.618 model $\left(\mathrm{R}_{001}=38.1 \mathrm{~mm} / \mathrm{h}\right)$; year 2016

\section{References}

[1] Rossi, T., De Sanctis, M., Ruggieri, M., Riva, C., Luini, L., Codispoti, G., Russo, E., Parca, G. "Satellite Communication and Propagation Experiments Through the Alphasat Q/V Band Aldo Paraboni Technology Demonstration Payload", IEEE Aerospace and Electronic Systems Magazine, 31(3), pp. 18-27, 2016. https://doi.org/10.1109/MAES.2016.150220

[2] Riva, C., Schönhuber, M., Codispoti, G., Martellucci, A., Capsoni, C., D’Amico, M., Luini, L., Resteghini, L., Nebuloni, R., Teschl, F., Cuervo, F., Plimon, K. "The Alphasat Aldo Paraboni scientific experiment: objectives and preliminary results", In: The 20th Ka and BroadBand Communications, Navigation and Earth Observation Conference, Vietri, Italy, 2014, pp. 347-355.

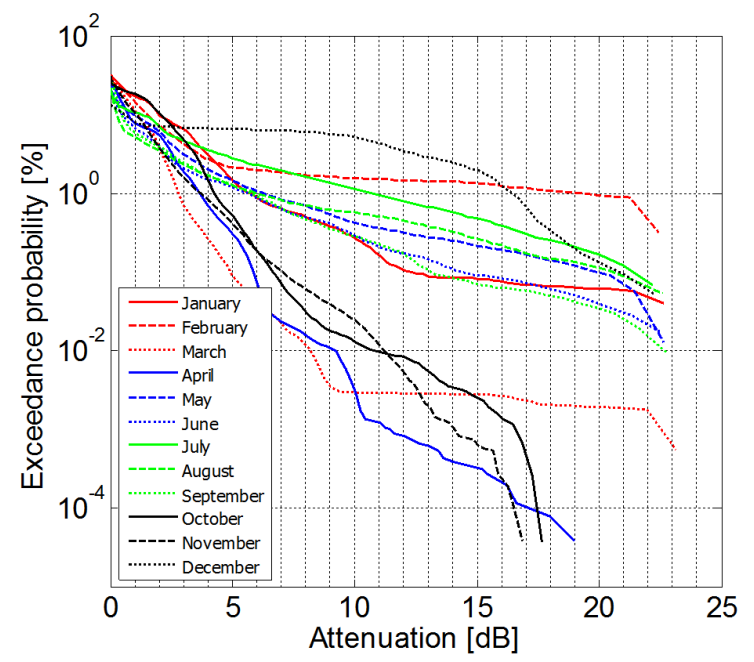

Fig. 11 CCDF of Ka and Q-band annual attenuation, 2016

\section{Conclusion}

In this paper we overviewed the Aldo Paraboni Scientific Experiment of the European Space Agency hosted by the Alphasat geosynchronous satellite. We introduced the European propagation measurement campaign performed by the Group of the AlphaSat Aldo Paraboni propagation Experimenters. A detailed description of the Budapest receiver station together with some measurement examples and yearly attenuation statistics are demonstrating the capabilities of the beacon signal receiver.

\section{Acknowledgement}

This research work was funded by the Government of Hungary through the ESA Contract 4000109841/13/NL/ KML under the PECS (Plan for European Cooperating States).

The authors would like to acknowledge the Agenzia Spaziale Italiana (A. S. I.) for supporting the Alphasat Aldo Paraboni propagation experiment.

[3] Teschl, F., Cuervo, F., Martellucci, A., Rivera Castro, J., Riva, C., Codispoti, G., Plimon, K., Schönhuber, M. "The Ka/Qband Alphasat Ground Propagation Terminal - First Months of Operation", In: 8th European Conference on Antennas and Propagation, Hague, The Netherlands, 2014, pp. 54-58. https://doi.org/10.1109/EuCAP.2014.6901691

[4] Machado, F., Pérez-Fontán, F., Pastoriza, V., Mariño, P. "The Kaand Q-band AlphaSat ground station in Vigo", In: 9th European Conference on Antennas and Propagation, Lisbon, Portugal, 2015, pp. 1-4.

[5] Riera, J. M., Siles, G. A., Garcia-del-Pino, P., Banarroch, A. "Alphasat Propagation Experiment in Madrid: Quality Assessment of the Measurements", In: 9th European Conference on Antennas and Propagation, Lisbon, Portugal, 2015, pp. 1-5. 
[6] Rocha, A., Jorge, F., Lima, J., Xavier, A. "Alphasat Q/V-band Propagation Campaign Preparation in Aveiro", In: 9th European Conference on Antennas and Propagation, Lisbon, Portugal, 2015, pp. $1-4$.

[7] Csurgai-Horváth, L., Rieger, I., Kertész, J. "The Aldo Paraboni Scientific Experiment: Ka/Q Band Receiver Station in Hungary", In: 9th European Conference on Antennas and Propagation, Lisbon, Portugal, 2015, pp. 1-6.

[8] Nessel, J., Zemba, M., Morse, J., Luini, L., Riva, C. "Preliminary Statistics from the NASA Alphasat Beacon Receiver in Milan, Italy", In: 9th European Conference on Antennas and Propagation, Lisbon, Portugal, 2015, pp. 1-5.

[9] Marziani, A., Consalvi, F., Chicarella, S., Restuccia, E., Amaduzzi, L., Marzano, F. S. "AlphaSat Ka-band and Q-band Receiving Station in Rome: Development, status and measurements", In: 9th European Conference on Antennas and Propagation, Lisbon, Portugal, 2015, pp. 1-5.

[10] Codispoti, G., Crino, V., Falzini, S., Russo, E., Vetrano, E. "Alphasat TDP5: AIT Activities on the Italian payload for communication and propagation experiments in $\mathrm{Q} / \mathrm{V}$ band", presented at 17th Ka and Broadband Communication Conference, Palermo, Italy, Oct. 3-5, 2011.

[11] Codispoti, G., Russo, E., Cornacchini, C., Falzini, S., Vernucci, A. "The Alphasat TDP\#5 Experiment and Challenges for Future Q/Vband Systems Exploitation", In: IET Seminar on Beyond Ka-Band: Meeting the Communication Bandwidth Requirements of the Future, Institute of Engineering and Technology (IET), London, United Kingdom, 2011, pp. 1-39.

https://doi.org/10.1049/ic.2011.0222
[12] COST Action IC0802, [online] Available at: http://www.tesa.prd. fr/cost [Accessed: 14 March 2018].

[13] 23rd Ka and Broadband Communications Conference, Trieste, Italy, Oct., 16-19, 2017.

[14] Recommendation ITU-R P.618-12, Propagation data and prediction methods required for the design of Earth-space telecommunication systems, ITU, 2015.

[15] Recommendation ITU-R P.837-6, Characteristics of precipitation for propagation modelling, ITU, 2012.

[16] Recommendation ITU-R P.838-3,Specific attenuation model for rain for use in prediction methods, ITU, 2005.

[17] Recommendation ITU-R P.839-4, Rain height model for prediction methods, ITU, 2013.

[18] Recommendation ITU-R P.678-3, Characterization of the variability of propagation phenomena and estimation of the risk associated with propagation margin, ITU, 2015.

[19] GRANTE Antenna Development and Production Corporation, HPA...380 Series Antenna Specifications, [online] Available at: http://grante.hu [Accessed: 14 March 2018].

[20] Totaltel Telecom Techniques Ltd., Point to point microwave radios, [online] Available at: http://www.totaltel.hu/en/index_en.html [Accessed: 14 March 2018].

[21] Recommendation ITU-R P.1511-1, Topography for Earth-space propagation modelling, ITU, 2015. 